\title{
Conocimientos y prácticas de las mujeres gestantes con relación a las zoonosis por animales de compañía
}

\author{
Knowledge and practices of pregnant women in relation to zoonoses by pets
}

\author{
Alejandra Garaycochea1, Daphne León ${ }^{2}$, Néstor Falcón ${ }^{2,3}$
}

\section{Resumen}

\begin{abstract}
El objetivo del estudio fue determinar conocimientos y prácticas relacionadas a zoonosis transmitidas por animales de compañía entre mujeres gestantes que se atendían en el Instituto Nacional Materno Perinatal de Lima, Perú. Se utilizó una encuesta que incluyó información demográfica de la persona encuestada, tenencia y manejo de animales de compañía, y conocimientos y prácticas potencialmente asociados a transmisión de zoonosis. Se recabaron 250 encuestas. El 87.2 y $46.8 \%$ de las gestantes mencionó tener canes y gatos, respectivamente, y el 93.6 y 94.9\% los utilizaban como animales de compañía, respectivamente. E1 91.6\% de las encuestadas manifestaron no conocer ni haber escuchado la palabra zoonosis; sin embargo, el $82.8 \%$ manifestaron saber que existen enfermedades que se transmiten de los animales a las personas. El 57.2\% de las encuestadas indicó que los animales de compañía pueden ocasionar problemas de salud durante el embarazo y el $44 \%$ consideró a los pelos como elemento de trasmisión de enfermedad. Las pr[acticas en relación con los animales de compañía variaron entre las encuestadas, antes y después de estar gestando. El conocimiento de las principales enfermedades zoonóticas potencialmente transmisibles a mujeres gestantes y su forma de transmisión fue baja, a excepción de la rabia.
\end{abstract}

Palabras clave: embarazadas; mordedura; rabia; zoonosis

\section{AbSTRACT}

The aim of this study was to determine knowledge and practices related to zoonoses transmitted by companion animals among pregnant women who were treated at the National Maternal and Perinatal Institute of Lima, Peru. A survey was used that included

\footnotetext{
${ }^{1}$ Grupo de Salud Pública Veterinaria (SAPUVET-PERÚ), Facultad de Medicina Veterinaria y Zootecnia, Universidad Peruana Cayetano Heredia, Lima, Perú

${ }^{2}$ Laboratorio de Epidemiología y Salud Pública en Veterinaria, Facultad de Medicina Veterinaria y Zootecnia, Universidad Peruana Cayetano Heredia, Lima, Perú

${ }^{3}$ E-mail: nestorfalcon@upch.pe
} 
demographic information of the person surveyed, possession and handling of pets, and knowledge and practices potentially associated with zoonosis transmission. A total of 250 surveys were collected. Results showed that 87.2 and $46.8 \%$ of pregnant women mentioned having dogs and cats, respectively, and 93.6 and $94.9 \%$ have them as pets, respectively. Also, $91.6 \%$ of respondents said they did not know or have heard the word zoonosis; however, $82.8 \%$ said they know that there are diseases that are transmitted from animals to people. Additionally, $57.2 \%$ of respondents indicated that pets can cause health problems during pregnancy and $44 \%$ considered hairs as an element of disease transmission. The practices in relation to pets varied among respondents before and after pregnancy. The knowledge of the main zoonotic diseases potentially transmissible to pregnant women and their form of transmission was low, except for rabies.

Key words: pregnant women; bite, rabies; zoonoses

\section{INTRODUCCIÓN}

Se denomina enfermedad zoonótica a cualquier enfermedad o infección que puede ser transmitida naturalmente de un animal vertebrado al humano (Fuentes et al., 2006) a través del contacto directo, inhalación, ingestión de agua o alimentos contaminados (Acero et al., 2014).

Existen grupos de personas que pueden ser más susceptibles a las zoonosis como son los niños, mujeres gestantes, ancianos y personas inmunocomprometidas con su salud (Ericson, 2013). En el caso de las mujeres gestantes, el cuerpo experimenta diversos cambios hormonales durante la gestación con el propósito de apoyar al crecimiento y desarrollo del feto. Diferentes estudios demuestran que la mayoría de las mujeres gestantes experimentan un debilitamiento del sistema inmune, lo cual puede generar un mayor riesgo de contraer enfermedades bacterianas o virales (Mor y Cárdenas, 2010). Algunos agentes infecciosos pueden aprovechar esto para producir enfermedades que pueden alterar o incluso llevar al aborto si no se toman las medidas sanitarias adecuadas (Jamieson et al., 2006).
Entre las enfermedades parasitarias consideradas de importancia para las gestantes se encuentra la toxoplasmosis ocasionada por Toxoplasma gondii (López et al., 2005; Restrepo, 2007), la equinococosis quística ocasionada por larvas de Echinococcus granulosus (Espinosa et al., 2012) y la toxocariasis causada por Toxocara canis y Toxocara cati (Magnaval et al., 2001). Entre las bacterianas se encuentran la leptospirosis producida por Leptospira spp (Shaked et al., 1993; Carles et al., 1995) y la bartonelosis o «enfermedad por arañazo de gato» causada por Bartonella henselae. A ellos se suma la rabia que es transmitida por el virus de la familia Rhabdoviridae (Swende y Achinge, 2009).

Al ser las mujeres gestantes una población particularmente susceptible de ser afectadas por enfermedades zoonóticas se hace necesario evaluar conocimientos y prácticas acerca de la transmisión de estas enfermedades para determinar conductas que puedan estar poniendo en riesgo a su descendencia o afectar el bienestar del animal de compañía. Por ello, el objetivo de estudio fue determinar conocimientos y prácticas relacionadas a zoonosis transmitidas por animales de compañía entre mujeres gestantes que se atendían en el Instituto Nacional Materno Perinatal de Lima, Perú. 
Cuadro 1. Grado de instrucción y ocupación de mujeres gestantes atendidas en el Instituto Nacional Materno Perinatal participantes del estudio. Lima 2017 ( $\mathrm{n}=250)$

\begin{tabular}{llcc}
\hline Variable & Estratos de la variable & $\mathrm{n}$ & $\%$ \\
\hline Grado de instrucción & Primaria incompleta & 1 & 0.4 \\
& Primaria completa & 3 & 1.2 \\
& Secundaria incompleta & 5 & 2.0 \\
& Secundaria completa & 142 & 56.8 \\
& Superior técnico & 63 & 25.2 \\
& Superior universitario & 36 & 14.4 \\
Ocupación & Ama de casa & 181 & 72.4 \\
& Profesional técnico & 21 & 8.4 \\
& Profesional universitario & 26 & 10.4 \\
& Otros & 8.8 \\
\hline
\end{tabular}

${ }^{1}$ Estudiante de educación técnico o superior (11), negocio o comercio (6), sin actividad definida (5)

\section{Materiales y Métodos}

El estudio transversal tipo prevalencia se desarrolló en el Instituto Nacional Materno Perinatal ubicado en el distrito de Cercado de Lima, Perú, entre setiembre y noviembre de 2017. La población objetivo estuvo compuesta por madres gestantes que se atendían en dicha institución durante el periodo de estudio.

Se realizaron encuestas dirigidas a mujeres gestantes que acudieron al establecimiento de salud para su control prenatal. El instrumento de recolección de información consideró variables de información demográfica de la encuestada, tenencia y manejo de animales de compañía y conocimientos y prácticas potencialmente asociados a transmisión de zoonosis.

La encuesta fue aplicada por el investigador principal. Cada entrevista duró entre 10 y 15 minutos y fue realizada en el horario de atención de consulta externa del establecimiento de salud. En compensación a la parti- cipación en el estudio, las entrevistadas recibieron un tríptico informativo acerca de las seis enfermedades zoonóticas de mayor importancia en el embarazo: bartonelosis, leptospirosis, toxocariasis, rabia, hidatidiosis y toxoplasmosis.

La información recogida en las encuestas fue transferida a una hoja del programa Microsoft Excel y analizada con el programa STATA 13.0. La variable cuantitativa (edad) fue resumida mediante media, desviación estándar y valores extremos. Las variables cualitativas se resumieron mediante cuadros de frecuencia. Las modificaciones de prácticas antes y después del embarazo se evaluaron mediante la prueba de Chi Cuadrado de McNemar.

El estudio se inició una vez que el Comité Institucional de Ética de la UPCH dio su aprobación mediante Constancia 537-1717. La participación en el estudio fue voluntaria y con previa firma de un consentimiento informado. En todo momento se salvaguardó el anonimato de las encuestadas. 
Cuadro 2. Conocimiento de las mujeres pacientes del Instituto Nacional Materno Perinatal sobre las principales enfermedades zoonóticas potencialmente transmisibles a mujeres gestantes. Lima $2017(\mathrm{n}=250)$

\begin{tabular}{lcccccc}
\hline Enfermedades & $\begin{array}{c}\text { No he escuchado de } \\
\text { esta enfermedad }\end{array}$ & $\begin{array}{c}\text { Si he escuchado, pero } \\
\text { no sé quién la } \\
\text { transmite }\end{array}$ & $\begin{array}{c}\text { Si he escuchado, lo } \\
\text { transmite... } \\
\text { (correcto) }\end{array}$ \\
\cline { 2 - 7 } & $\mathrm{n}$ & $\%$ & $\mathrm{n}$ & $\%$ & $\mathrm{n}$ & $\%$ \\
\hline Rabia & 2 & 0.8 & 23 & 9.2 & 225 & 90.0 \\
Toxoplasmosis & 192 & 76.8 & 19 & 7.6 & 39 & 15.6 \\
Toxocariasis & 235 & 94.0 & 9 & 3.6 & 6 & 2.4 \\
Leptospirosis & 237 & 94.8 & 8 & 3.2 & 5 & 2.0 \\
Bartonelosis & 238 & 95.2 & 10 & 4.0 & 2 & 0.8 \\
Hidatidosis & 240 & 96.0 & 8 & 3.2 & 1 & 0.4 \\
\hline
\end{tabular}

\section{Resultados}

El estudio recabó 250 encuestas de mujeres gestantes. La mayoría de ellas contaba con secundaria completa y la principal ocupación mencionada fue la de ama de casa (Cuadro 1). Las encuestadas tenían $30.1 \pm$ 5.7 años (Mínimo: 18; Máximo: 43.3 años). Del total de gestantes, el $87.2 \%$ (218) mencionó tener canes y, de ellas, el 93.6\% (204) lo utilizaban como animal de compañía. En el caso de los gatos, el 46.8\% (117) indicó criar a esta especie animal, de los cuales, el $94.9 \%$ (111) eran considerados como animales de compañía.

El 98.2\% (214/218) de las encuestadas que poseían canes y el 53.8\% (63/117) de quienes poseían gatos indicaron que vacunan anualmente contra la rabia a sus animales. En el momento de la encuesta, las entrevistadas mencionaron que el $35.3 \%(77 / 218)$ y el $29.4 \%(64 / 218)$ de los canes y el $65.0 \%$ (76/117) y el 59.0\% (69/117) de los gatos no habían recibido tratamiento contra parásitos internos o externos, respectivamente, en los últimos seis meses o nunca.
De las 218 encuestadas que mencionaron tener perros, el 7.8\% (17) manifestaron que alguno de sus perros se encontraba esterilizado/castrado y el 8.3\% (18) que todos lo estaban. En el caso de las 117 encuestadas que mencionaron tener gato, el 5.2\% (13) y $6.0 \%$ (15) manifestaron tener algunos y todos los gatos esterilizados/castrados, respectivamente.

El 46.8\% (102) de las gestantes con canes mencionó que la alimentación de los animales era mixta, el 35.3\% (77) les daba un balanceado y el $17.9 \%$ (39) les daba comida casera. El 77.5\% (169) de las encuestadas realizaban baños a sus canes por lo menos una vez al mes y el lugar de las deposiciones era en los parques o en la vía pública para el 47.2\% (103) y en el patio, jardín o techo de la vivienda del propietario para el $44.5 \%$ (97). El resto de las encuestadas no contestó la pregunta. En el caso de las gestantes con gato, el 61.5\% (72) mencionó que los alimentaban con balanceado, 22.2\% (26) con balanceado y comida casera y solo $16.2 \%$ (19) los alimentaba exclusivamente con comida casera. El 28.2\% (33) realizaban baños a sus gatos por lo menos una vez al 
Cuadro 3. Conocimientos sobre animales y causas que pueden ocasionar enfermedad en gestantes según opinión de las mujeres encuestadas. Instituto Nacional Materno Perinatal. Lima $2017(\mathrm{n}=250)$

\begin{tabular}{llcc}
\hline Variable & Estrato de la variable & $\mathrm{n}$ & $\%$ \\
\hline Animal que produce & Gato & 143 & 57.2 \\
alteraciones en la salud & Perro & 3 & 1.2 \\
& Varios (gato-perro-otros ${ }^{1}$ ) & 24 & 9.6 \\
& No sabe & 18 & 7.2 \\
& Ninguno & 62 & 24.8 \\
Causa que produce las & Pelos & 110 & 44.0 \\
alteraciones de la salud & Parásitos (sin especificar) & 7 & 2.8 \\
& Toxoplasmosis & 6 & 2.4 \\
& Otros & 11 & 4.4 \\
& Pelos/parásitos (sin especificar) & 20 & 8.0 \\
& Pelos/otros & 3.6 \\
& Pelos/toxoplasma & 9 & 0.4 \\
& No contestó & 1 & 34.4 \\
\hline
\end{tabular}

${ }^{1}$ Cualquier otro animal

${ }^{2}$ Enfermedad sin definir, suciedad, orina, deposiciones, hongos, ácaros

mes y el lugar de las deposiciones era en los parques o vía pública para el $34.2 \%$ (40), en cajas de arena para el $30.8 \%$ (36) y en el patio, jardín o techo de la vivienda del propietario para el 29.9\% (35). El resto de las encuestadas no contestó la pregunta.

Solo el 8.4\% (21) de las encuestadas manifestaron conocer o haber escuchado la palabra zoonosis y de ellos, el $28.6 \%$ (6) lo definieron correctamente, lo que representó solamente el $2.4 \%$ del total de encuestadas (6/250). Los medios de información de donde habrían recibido la información fueron Internet, hospital, televisión o universidad. El $82.8 \%$ (207) manifestó saber que existen enfermedades que se transmiten de los animales a las personas. El conocimiento de las principales enfermedades zoonóticas potencialmente transmisibles a mujeres gestantes y su forma de transmisión fue baja, a excepción de la rabia (Cuadro 2).
En cuanto a los animales que pueden ocasionar problemas de salud durante el embarazo, se mencionó principalmente a los gatos y como causa específica se señaló a los pelos del gato (Cuadro 3). Las prácticas en relación con los animales de compañía variaron entre las encuestadas, antes y después de estar gestantes. Las proporciones de respuestas afirmativas fueron significativamente menores después de la gestación en comparación con las respuestas antes de haber quedado gestantes $(\mathrm{p}<0.001)$, a excepción de la práctica de deshacerse del animal de compañía (Cuadro 4).

El 13.6\% (34/250) manifestó que dejaría que el animal de compañía lama a su bebé. El 23.6\% (59) aislaría a los animales del bebé (lo daría en adopción, lo aislaría en un espacio de la casa, etc.), el 18.4\% (46) dejaría que su bebé juegue con los animales, el 78.8\% (197) afirmó que estaría de acuerdo con te- 
Cuadro 4. Prácticas de las mujeres gestantes relacionadas con los animales de compañía antes y después de conocer que se encontraban en gestación. Instituto Nacional Materno Perinatal. Lima $2017(\mathrm{n}=250)$

\begin{tabular}{lcccc}
\hline \multirow{2}{*}{$\begin{array}{l}\text { Práctica en relación con animales de } \\
\text { compañía }\end{array}$} & \multicolumn{2}{c}{ Antes } & \multicolumn{2}{c}{ Después } \\
\cline { 2 - 5 } & $\mathrm{n}$ & $\%$ & $\mathrm{n}$ & $\%$ \\
\hline Beso al animal & 82 & $32.8^{\mathrm{a}}$ & 31 & $12.4^{\mathrm{b}}$ \\
Caricias al animal & 232 & $92.8^{\mathrm{a}}$ & 170 & $68.0^{\mathrm{b}}$ \\
Dormir con el animal & 53 & $21.2^{\mathrm{a}}$ & 5 & $2.0^{\mathrm{b}}$ \\
Limpiar la cama del animal & 177 & $70.8^{\mathrm{a}}$ & 102 & $40.8^{\mathrm{b}}$ \\
Limpieza de heces del animal & 162 & $64.8^{\mathrm{a}}$ & 96 & $38.4^{\mathrm{b}}$ \\
Jugar con el animal & 221 & $88.4^{\mathrm{a}}$ & 160 & $64.0^{\mathrm{b}}$ \\
Sacar a pasear al animal & 143 & $57.2^{\mathrm{a}}$ & 78 & $31.2^{\mathrm{b}}$ \\
Deshacerse del animal & 18 & $7.2^{\mathrm{a}}$ & 24 & $9.6^{\mathrm{a}}$ \\
\hline
\end{tabular}

a,b Letras diferentes indican diferencia significativa entre la opinión antes y después para la práctica en evaluación $(p<0.001)$

ner un animal de compañía a solicitud del hijo y el 76\% (190) estaría dispuesta a adoptar un animal de la calle.

\section{Discusión}

La mayoría de las mujeres gestantes consideraba vacunar a los perros contra la rabia. Esto se justificaría debido a que el Ministerio de Salud (MINSA) se encarga de difundir las campañas gratuitas de vacunación antirrábica de canes (VANCAN) y realiza esfuerzos para hacer cumplir la norma técnica de salud para la prevención y control de la rabia humana (MINSA, 2017). La vacunación antirrábica de gatos no es necesaria en la ciudad de Lima, debido a que esta especie no se encuentra expuesta directamente a la enfermedad, habiéndose demostrado que el control de la rabia en perros es suficiente para el control de la rabia en la población de gatos (Málaga, 2010). A pesar de ello, más del 50\% de las entrevistadas habría vacunado a sus gatos contra la rabia.
El manejo de la desparasitación interna cada tres meses es reconocido por solo un tercio de las encuestadas, por lo que se puede considerar que hay una población de animales que no son desparasitados con la frecuencia que se requiere para mantener una condición libre de parasitosis, tanto en perros como en gatos (Park et al., 2017). Se debe tener en cuenta que se ha demostrado que la desparasitación con una frecuencia de 1-3 veces por año no ofrece garantía de suficiente protección (Pereira y Pérez, 2002). Con respecto a las desparasitaciones externas, se debe considerar la presencia de Dipylidium caninum, parásito que usa como hospedero intermediario a las pulgas y al perro o gato como hospedero definitivo (Wani et al., 2015), desde un punto de vista epidemiológico y clínico (Szwaja et al, 2011), a pesar de que las infecciones por $D$. caninum en humanos son relativamente raras y afectan particularmente a niños.

En cuanto a la práctica de alimentación de los animales de compañía, la mayoría incluyó alimento casero, que en el caso de los 
perros, puede abarcar desde sobras de las comidas hasta alimento preparado o vísceras. Un estudio realizado en zona urbana de Pasco (Perú) en la que se aceptaba múltiples respuestas, reportó que el $90.9 \%$ alimentaba a sus perros con sobras del almuerzo, $31.7 \%$ le preparaba su alimento y $14.3 \%$ le daba vísceras crudas (Núñez et al., 2003). Este resultado es relevante ya que de esa manera se puede favorecer la diseminación del parásito Equinococcus granulosus. En caso de las gestantes, el aumento de la presión provocada por el útero grávido puede ocasionar la rotura de un quiste hidatídico o este puede inducir a un parto prematuro debido al espacio que ocupa. La rotura del quiste hidatídico durante el parto puede provocar una reacción anafiláctica, e incluso puede provocar la muerte de la madre y el feto (Espinosa et al., 2012).

En el caso de gatos, la alimentación predominante era el concentrado o alimento balanceado. La alimentación casera, que es una práctica riesgosa, tuvo una baja frecuencia en el estudio. Se debe tener en cuenta que los gatos se pueden infectar con Toxoplasma gondii, mayormente tras la ingestión de quistes que se encuentran en la carne de los hospederos intermediarios como roedores, palomas, o mediante el consumo de alimentos o agua contaminada con quistes. Los gatos eliminan los ooquistes al ambiente durante un corto periodo de tiempo en su vida (Harvey y Taskar, 2014).

El $46.8 \%$ de las encuestadas indicaron que el lugar de deposiciones de sus perros era en el parque o vía pública. Esta práctica favorece la contaminación ambiental exponiendo a las personas, entre ellas a las mujeres gestantes, a diversas formas parasitarias, incluyendo la toxocariasis. Se ha reportado que el $30 \%$ de parques públicos de distritos del Cono Sur, $41 \%$ en el Cono Este, $34 \%$ en el Cono Norte y $63 \%$ en el Cono Oeste de la ciudad de Lima se encuentran contaminados con huevos o larvas de T. canis (Chávez et al., 2002). En caso de la defecación en el hogar se debe de tener cuidado en eliminar los restos de forma higiénica. En una evaluación de hogares, donde se recolectó 120 muestras de los suelos de las viviendas, 11 resultaron positivas $(9.2 \%)$ correspondiendo a los parásitos D. caninum, $T$. canis, Taenia sp y Ascaris sp (Guillinta, 2011).

Resultados de estudios en humanos han demostrado que la toxocariosis es una enfermedad zoonótica frecuente (Espinoza et al., 2003), pudiendo presentarse como «larva migratoria visceral» o como «larva migratoria ocular». En ambas formas se presentan signos clínicos evidentes (Despommier, 2003). En los niños, la infección visceral es la más común y puede causar distintos síntomas que persisten entre 6 y 24 meses. La infección ocular puede causar pérdida de la visión (Barriga, 1988).

La práctica de permitir orinar en el hogar (patio, jardín o techo) podría favorecer la exposición de las gestantes a enfermedades como leptospirosis si es que los canes no están vacunados contra la enfermedad y se encuentran desarrollándola. La vía de infección de la Leptospira es por exposición directa $o$ indirecta a la orina de un animal infectado (Bolin, 2002).

Toxoplasma gondii requiere de condiciones ambientales específicas para su supervivencia, encontrándose mayormente en regiones cálidas $\mathrm{y} / \mathrm{o}$ húmedas (como la ciudad de Lima), y en menor grado en climas secos y fríos. Cuando los ooquistes encuentran condiciones favorables en el ambiente externo alcanzan su estado infectante en 1-3 días (Cerro et al., 2009). Es por esto que las excretas se deben remover diariamente de la caja de arena con guantes y realizar el lavado de las manos posteriormente a la limpieza (Basso y Venturini, 2008).

El consumo de quistes tisulares de $T$. gondii en los tejidos animales para consumo humano o mediante la presencia de ooquistes en frutas y verduras son las principales formas de transmisión de la enfermedad al humano. Se estima que el $72 \%$ de la carne de 
cordero, $28 \%$ de cerdo, $9 \%$ de equino y $4 \%$ de res que es comercializada contienen estas formas infectantes (Grandía et al., 2013). En Colombia se ha encontrado que estas vías pueden ser la fuente de infección para el 25\% de los casos de toxoplasmosis durante el embarazo, especialmente a través de la carne de cerdo (Franco, 2015). La toxoplasmosis se presenta usualmente de forma asintomática; sin embargo, en personas inmunosuprimidas puede producir graves complicaciones y muerte, al igual que en los hijos de las mujeres que adquieren la infección primaria durante la gestación (Restrepo, 2007). Las consecuencias para el bebé en mujeres embarazadas incluyen hidrocefalia, microencefalia, calcificaciones cerebrales, coriorretinitis, y aborto (López et al., 2005).

El conocimiento del término zoonosis y su significado fue bajo entre las mujeres gestantes, siendo similar al estudio de Tarabla y Fernández (2009) en la Provincia de Santa $\mathrm{Fe}$ (Argentina) con amas de casa. En todo caso, más importante que conocer el significado del término zoonosis, debe ser conocer la existencia de enfermedades que pueden transmitirse de animales a las personas y principalmente las formas de transmisión. E1 90\% de las mujeres gestantes han escuchado y saben qué animal puede transmitir la rabia, debido a las campañas anuales el control de esta enfermedad. En el estudio de Llalla y Falcón (2014) en instituciones educativas de tres estratos socioeconómicos de Lima se encontró que el $89.3 \%$ de escolares de cuarto y quinto año de secundaria reconocieron haber escuchado acerca de la rabia y $78.5 \%$ indicaron correctamente su forma de transmisión. Por otra parte, el 15.6\% de las mujeres gestantes que reconocieron que los animales pueden transmitir la toxoplasmosis concuerda con resultados del estudio de Llalla y Falcón (2014).

El conocimiento del agente etiológico, así como la forma de transmisión de la hidatidiosis, bartonelosis, leptospirosis y toxocariasis estuvo por debajo del 3\% por parte de las mujeres gestantes encuestadas, resultado similar a lo encontrado en otros grupos poblacionales. Por ejemplo, Molineri et al. (2014) concluyen que la toxocariasis, la hidatidosis, la leptospirosis y la toxoplasmosis fueron escasamente reconocidas por los trabajadores rurales. Astaiza et al. (2014) mencionan que existe un conocimiento menor de las enfermedades causadas por parásitos como la cisticercosis $(11.59 \%)$ y la toxocariosis (10.64\%). Para lograr que la población obtenga el grado de conocimiento que permita mejorar la prevención de estas parasitosis se deben utilizar los medios de comunicación masivos y hacer uso de las instituciones educativas. En caso de estas últimas, el uso de herramientas educativas lúdicas podría representar una alternativa para promover la salud entre la población escolar (Mejía et al., 2017).

Las encuestadas indicaron que el gato y el pelo eran las potenciales causas de producir alteraciones en su salud durante la gestación, lo cual demuestra que no existe un conocimiento correcto acerca de las vías de transmisión de las enfermedades zoonóticas. Por ello, una adecuada campaña de educación sanitaria en esta población podría mejorar los conocimientos respecto a las formas de transmisión, impacto sobre la salud humana y animal y forma de prevención de las enfermedades que son de importancia en la población a intervenir.

El manejo y trato con los animales antes y después de conocer que las encuestadas se encontraban en gestación muestran comportamientos dirigidos a un menor contacto con los animales. La práctica de besar al perro conlleva al contacto con bacterias que se encuentran en la cavidad oral de los perros (Martínez, 2005; Low y Greenwood, 2008). Si bien la limpieza de la cama de los animales es una práctica importante y necesaria en el hogar, la responsabilidad debe asumirla otro miembro de la familia y no la mujer gestante. La práctica de pasear a los perros también disminuyó una vez conocida la condición de estar gestante. En este caso, si bien es conveniente limitar el contacto con los animales, 
disminuir la práctica del paseo puede favorecer la persistencia de malestares propios de la gestación como dolores de espalda o en la parte baja del vientre, calambres en las piernas, que el reposo no ayuda a superar (Gallo et al., 2016).

Se debe de tener en cuenta que las enfermedades zoonóticas actualmente han cobrado un mayor impacto debido a la estrecha relación que existe entre las personas y los animales. Al ser las madres gestantes un grupo especialmente vulnerable, estas se pueden encontrar expuestas a contraer distintas enfermedades. Por ello la tenencia no responsable de animales de compañía puede conllevar a alterar la convivencia de ellas con las personas y generar problemas a la salud de las mujeres gestantes debido al desconocimiento de las principales enfermedades zoonóticas y su forma de transmisión. Además el desconocimiento puede llevar a prácticas que pueden afectar el bienestar animal. Por ello se hace necesario desarrollar campañas educativas que permitan mantener una relación equilibrada entre las gestantes y animales a fin de que la convivencia se mantenga para mutuo beneficio de ambas poblaciones.

\section{Literatura Citada}

1. Acero PV, Gil TD, Gutiérrez VE, Porto P. 2014. Salud pública, responsabilidad social de la medicina veterinaria y la tenencia responsable de mascotas: una reflexión necesaria. REDVET 15(5). [Internet]. Disponible en: http:// www.veterinaria.org/revistas/redvet/ n050514B.html

2. Astaiza-Martínez JM, BenavidesMelo CM, Vallejo-Timarán DA. 2014. Evaluación del conocimiento sobre enfermedades zoonóticas en estudiantes de bachillerato de instituciones educativas del sector rural del municipio de Pasto, Nariño. Vet Zootec 8: 110-119.
3. Barriga O. 1988. A critical look at the importance, prevalence and control of toxocariasis and the possibilities of immunological control. Vet Parasitol 29: 195-234. doi: 10.1016/0304-4017(88)90126-4

4. Basso WU, Venturini MC. 2008. La toxoplasmosis en los animales domésticos y silvestres criados en cautiverio. En: Cacchione RA, Durlach R, Martino P (eds). Temas de zoonosis IV. Buenos Aires, Argentina: Ed. Asociación Argentina de Zoonosis. p 355-361.

5. Bolin C. 2002. Leptospirosis posing new threat for canine hepatic, renal disease. In Focus June 2002: 31-34.

6. Carles G, Montoya E, Joly F, Peneau C. 1995. Leptospirosis and pregnancy. Eleven cases in French Guyana. J Gynecol Obst Bio R 24: 418-421.

7. Cerro T, Chávez V, Casas A, Suárez A, Rubio V. 2009. Frecuencia de Toxoplasma gondii en gatos de Lima Metropolitana y concordancia entre las técnicas de inmunofluorescencia indirecta y hemaglutinación indirecta. Rev Inv Vet Perú 20: 285-290. doi: 10.15381/ rivep.v20i2.624

8. Chávez A, Casas E, Serrano M, Cajas J, Verde J, La Rosa V, López J. 2002. Riesgo de contraer enfermedades parasitarias en los parques públicos de Lima y Callao. Rev Inv Vet Perú 13: 84-91. doi: 10.15381/rivep.v13i2.7337

9. Despommier D. 2003. Toxocariasis: clinical aspects, epidemiology, medical ecology, and molecular aspects. Clin Microbiol Rev 16: 265-272. doi: 10.1128/ CMR.16.2.265-272.2003

10. Ericson C. 2013. The control of notifiable zoonotic diseases in pet animals in Sweden. PhD thesis. Budapest: Szent István University. $50 \mathrm{p}$.

11. Espinosa J, Jover R, Ramírez, R, Muci T, Lluís F. 2012. Quiste hidatídico hepático durante el embarazo. Cirugía Española 90: 63-65. doi: 10.1016/j.ciresp.2010.07.019 
12. Espinoza Y, Huapaya P, Sevilla C, Huiza A, Jiménez S, Náquira C. 2003. Toxocariosis humana: seroprevalencia en población de Lima mediante la técnica de ELISA. An Fac Med 64: 228-232.

13. Franco E. 2015. Prevalencia y factores asociados a la infección por Toxoplasma gondii en carne procedente de plantas de beneficio animal con destino nacional. Tesis Doctoral. Bogotá: Univ. Nacional de Colombia. 84 p.

14. Fuentes M, Pérez L, Suárez, Y, Soca M, Martínez A. 2006. La zoonosis como ciencia y su impacto social. REDVET 7(9). [Internet]. Disponible en: h t t p : / / w w w.redaly c.org/ articulo.oa? $\mathrm{id}=63612675013$

15. Gallo-Padilla D, Gallo-Padilla C, GaIlo-Vallejo FJ, Gallo-Vallejo JL. 2016. Lumbalgia durante el embarazo. Abordaje multidisciplinar. SEMERGEN 42: 59-64. doi: 10.1016/j.semerg.2015.06.005

16. Grandía G, Entrena G, Cruz H. 2013. Toxoplasmosis en Felis catus: etiología, epidemiología y enfermedad. Rev Inv Vet Perú 24: 131-149. doi: 10.15381/ rivep.v24i2.2469

17. Guillinta Y. 2011. Presencia de huevos de Toxocara spp en el suelo de ambientes frecuentados por niños en edad escolar. Tesis de Médico Veterinario Zootecnista. Lima: Univ. Peruana Cayetano Heredia. $27 \mathrm{p}$.

18. Harvey A, Tasker S. 2014. Manual de medicina felina. Barcelona: Ed Lexus. $720 \mathrm{p}$.

19. Jamieson DJ, Theiler RN, Rasmussen $S A$. 2006. Emerging infections and pregnancy. Emerg Infect Dis 12: 16381643. doi: $10.3201 /$ eid1211.060152

20. Llalla H, Falcón N. 2014. Conocimientos acerca de las principales enfermedades zoonóticas del Perú entre escolares limeños que terminan estudios secundarios. Rev Cien Vet 30(2). [Internet]. Disponible en: http://cmvl.pe/wp-content/ uploads/2014/06/MV-30-2.pdf

21. López-Castillo C, Díaz-Ramírez J, Gómez-Marín J. 2005. Factores de riesgo en mujeres embarazadas, infec- tadas por Toxoplasma gondii en Armenia-Colombia. Rev Salud Pública. 7: 180-190. doi: 10.1590/S012400642005000200006

22. Low SC, Greenwood JE. 2008. Capnocytophaga canimorsus: infection, septicaemia, recovery and reconstruction. J Med Microbiol 57: 901-903. doi: 10.1099/jmm.0.47756-0

23. Magnaval JF, Glickman LT, Dorchies P, Morassin B. 2001. Highlights of human toxocariasis. Korean J Parasitol 39: 1-11. doi: 10.3347/kjp.2001.39.1.1

24. Málaga H. 2010. Epidemiología veterinaria. Lima: Univ. Peruana Cayetano Heredia. $183 \mathrm{p}$.

25. Martínez MA. 2005. Microorganismos asociados a infecciones por mordeduras de perros y gatos. Mon Electr Patol Vet 2(1): 1-16.

26. Mejía CR, Vicuña F, Carpio Y, León D. 2017. Actividades y herramientas lúdicas en educación y promoción de la salud. Rev Cien Vet 33: 5-11.

27. [MINSA] Ministerio de Salud. (2017). Norma técnica de salud para la prevención y control de la rabia humana en el Perú. Lima: MINSA. 131 p. [Internet]. Disponible en: http://bvs.minsa.gob.pe/ local/DGSP/770_DGSP242.pdf

28. Molineri AI, Signorini ML, Tarabla HD. 2014. Conocimiento de las vías de transmisión de las zoonosis y de las especies afectadas entre los trabajadores rurales. Rev Argent Microbiol 46: 7-13. doi: 10.1016/S0325-7541(14)70041-0

29. Mor G, Cardenas I. 2010. The immune system in pregnancy: a unique complexity. Am J Reprod Immunol 63: 425-433. doi: 10.1111/j.1600-0897.2010.00836.x

30. Núñez E, Calero D, Estares L, Morales A. 2003. Prevalencia y factores de riesgo de hidatidosis en población general del distrito de Ninacaca-Pasco, Perú 2001. An Fac Med 64: 34-42. doi: 10.15381/anales.v64i1.1419

31. Park M, Road G, Worcs M. 2017. Worm control in dogs and cats. European Scientific Counsel Companion Animal Parasites. Guideline 01. $3^{\text {rd }}$ ed. [Internet]. 
Available in: http://www.esccap.org/ uploads/docs/0x0o7jda_ESCCAP_Guideline_01_Third_Edition_July_2017.pdf

32. Pereira A, Pérez M. 2002. Toxoplas-mosis. Ámbito farmacéutico. Offarm 22: 123-129.

33. Restrepo M. 2007. Toxoplasmosis: zoonosis parasitaria. Rev CES Med 21: 41-48.

34. Shaked Y, Shpilberg O, Samra D, Samra Y. 1993. Leptospirosis in pregnancy and its effect on the fetus: case report and review. Clin Infect Dis 17: 241-243. doi: 10.1093/clinids/17.2.241

35. Swende TZ, Achinge GI. 2009. Clinical rabies in pregnancy with delivery of a live fetus: a case report. Niger J Med 18: 114-115.
36. Szwaja B, Romañski L, Zabczyk M. 2011. A case of Dipylidium caninum infection in a child from the southeastern Poland. Wiad Parazyt 57: 175-178.

37. Tarabla HD, Fernández G 2009. Conocimiento sobre zoonosis en amas de casa del ámbito urbano de la ciudad de Coronda, Santa Fe. FAVE 8: 11-14. doi: 10.14409/favecv.v8i1.1475

38. Wani ZA, Allaie IM, Shah BM, Raies A, Athar H, Junaid S. 2015. Dipylidium caninum infection in dogs infested with fleas. J Parasit Dis 39: 73 75. doi: 10.1007/s12639-013-0281-x 